\title{
A compensation method for the hysteresis error of DVD VCM
}

\author{
Chih-Liang Chu ${ }^{1}$, Kuang-Chao Fan ${ }^{2,3}$ and Ye-Jing Chen ${ }^{2}$ \\ ${ }^{1}$ Department of Mechanical Engineering, Southern Taiwan University of Technology, \\ Tainan, Taiwan, Republic of China \\ ${ }^{2}$ School of Instrumentation, Hefei University of Technology, Hefei, \\ People's Republic of China \\ ${ }^{3}$ Department of Mechanical Engineering, National Taiwan University, Taipei, Taiwan, \\ Republic of China \\ E-mail: cliang@mail.stut.edu.tw
}

Received 3 October 2003, in final form 7 January 2004

Published 19 March 2004

Online at stacks.iop.org/MST/15/734 (DOI: 10.1088/0957-0233/15/4/017)

\begin{abstract}
The present study considers an autofocusing laser probe system used for the measurement of the surface profile and roughness of an object. The system is based upon a modified pickup head of a commercially available DVD player which uses a voice coil motor (VCM) to drive an objective lens during the autofocusing process. It is known that hysteresis of the VCM during the autofocusing process reduces the precision of the measurement results. Consequently, the present investigation adopts a hysteresis model to develop a compensation method which can improve the accuracy of the optical pickup head within the measurement system.
\end{abstract}

Keywords: DVD pickup head, autofocus, voice coil motor, hysteresis

\section{Introduction}

In considering the relative advantages and disadvantages of contact and non-contact type probes, previous researchers have concluded that optical non-contact type probes are more suitable for the measurement of soft, thin or micro-scale components since, by definition, they do not come into contact with the component surface and, therefore, there is no possibility of the surface being damaged $[1,2]$. Furthermore, since there is no surface contact force, measurement errors induced by surface deformation can be avoided. Additionally, the laser probe radius is very small and, hence, there is no need to compensate for the radius probe effect when determining the actual surface measurement values. The inherent benefits of the non-contact type probe have led to their increasing adoption for measurement purposes within the ultra-precision fabrication field. Simultaneously, considerable attention has been devoted to developing techniques in which laser technology can be used to access data, e.g. in such devices as CD or DVD players. The light routing and detection capabilities of these devices, together with their autofocusing characteristics, render them suitable for use in optical measurement systems. Nowadays, the optical pickup technology within commercially available DVD players is well developed, and is comparatively inexpensive. Accordingly, a review of the related literature reveals that many researchers have applied the autofocusing components of the commercial optical pickup head within high-precision measurement applications since the early 1990s. Several of these studies have concentrated specifically upon surface profile measurement. For example, Benschop and Rosmalen [3] developed a scanning optical microscope in which the laser diode light source of a CD pickup head was replaced by a high power laser directed by a fibre optics arrangement. A confocal photodiode was located in the vicinity of the laser source, and the object surface condition was scanned by sensing the phase shift of the light reflected from the surface. Furthermore, the proposed system incorporated a four-quadrant photodiode and an autofocusing function in order to facilitate the profile measurement of an object.

Armstrong and Fitzgerald [4] modified the laser head of a CD player in order to develop a low cost autocollimator. In their approach, the objective lens was removed from the pickup head so that the light reflected from the measured object could fall directly upon the four-quadrant photodiode. The voltage difference between the paired photodiodes was then used to interpolate the corresponding angle changes in two perpendicular directions. It was shown that the developed 
system had an effective measurement range of $1200 \mu \mathrm{rad}$ and a resolution of $\pm 0.006 \mu \mathrm{rad}$.

Quercioli et al [5] employed a laser pickup head to develop a velocimeter, in which the original laser beam emitted from the laser diode was separated into three beams by means of a diffraction grating and then finally fell upon the surface of the disk to be measured. Given that the distance between two light beams was known, by measuring the time interval between two reflected light beams, the proposed velocimeter was capable of determining the rotational speed of the measured object.

Zhang and Cai [6] developed an autofocusing surface roughness measurement system in which the VCM was locked in place within the pickup head, and a piezoelectric translator (PZT) was used in its place to drive the pickup head during the autofocusing operation. However, the high voltage supply required to drive the PZT device served as a deterrent to its widespread application. Furthermore, its operational measurement range was limited to a distance of approximately $30 \mu \mathrm{m}$.

In a previous study [7], the current authors also locked the voice coil motor in a CD pickup head and treated the entire head unit as a focusing probe. Under the proposed approach, a precise surface profile measurement was obtained by applying the one-to-one nature of the relationship between the FES magnitude and the focus error signal (FES) in the Scurve domain. It was shown that the developed system had a measurement accuracy of $34 \mathrm{~nm}$ and an effective measurement range of $10 \mu \mathrm{m}$.

In a subsequent study, the current authors [8] developed a low cost, high-precision optical autofocusing probe measurement system which used an analogue PD controller to drive the VCM so that the effective measuring range could extend to several hundred micrometres. However, it was determined that the measurement precision of the proposed system was degraded by the hysteresis effect of the VCM. Consequently, it is the aim of the present investigation to analyse the VCM hysteresis effect within the optical pickup head, and to develop an effective hysteresis compensation method. A hysteresis modelling approach is adopted to compensate for the hysteresis error so as to improve the accuracy and performance of the optical pickup head within the autofocusing laser probe measurement system. The present study retains the use of the VCM within the pickup head to drive the objective lens, i.e. rather than using a PZT, in order to minimize the power supply voltage requirements, and to provide a reasonable measurement range in the order of several hundred micrometres.

A review of the published literature indicates that most previous researchers have concentrated their efforts upon the hysteresis compensation of PZT devices, and that comparatively few studies have addressed the issue of VCM hysteresis compensation. The mathematical hysteresis models presented within these studies may be divided into the following three broad categories. (1) The generalized Maxwell model [9], in which the nonlinear piezoelectric hysteresis effect is modelled by means of a vibration system comprising a zero mass block, a linear spring and an external friction damping arrangement. The relationship between the supply voltage and the corresponding hysteresis displacement is represented by the relationship between the displacement

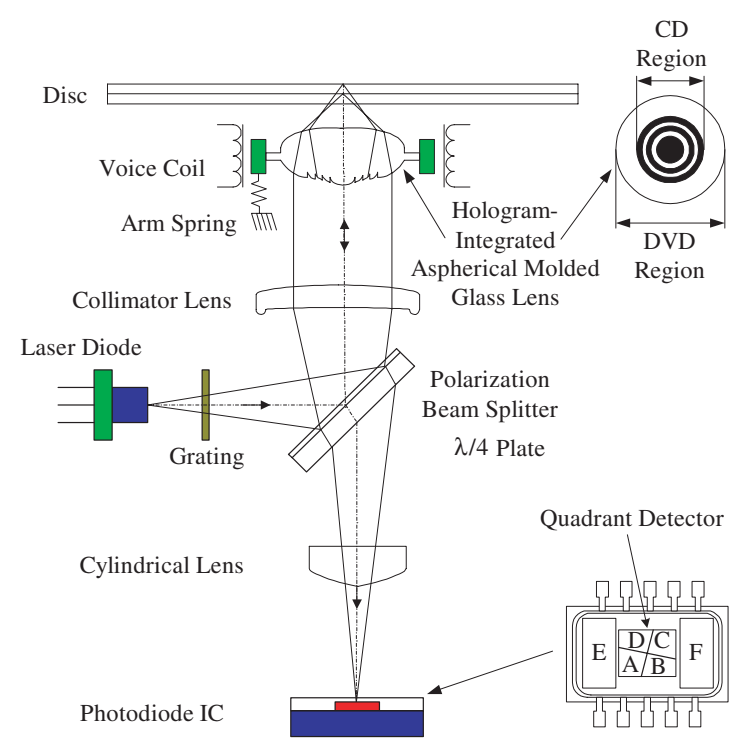

Figure 1. Internal structure of the DVD pickup head.

of the zero mass block and linear spring and the applied force. (2) The approximated polynomial method [10], which approximates the upper and lower hysteresis curves by means of a polynomial expression. (3) The Preisach model [11], which describes the nonlinear behaviour of the hysteresis phenomenon in ferromagnetic materials. This model has previously been adopted by many researchers, and has proved to be an effective means of describing the hysteresis phenomenon. It has been shown that the use of this model to simulate the hysteresis curves of a PZT device yields precise results. Accordingly, the present study chooses to apply this model to simulate the hysteresis behaviour of a VCM, and then to develop an efficient hysteresis compensation method. This report would be the first one ever successfully compensated the hysteresis error of the DVD pickup VCM using the Preisach model.

\section{Analysis of the autofocusing laser probe hysteresis phenomenon}

\subsection{DVD pickup head operation}

The general arrangement of the DVD pickup head is presented in figure 1. A laser diode device is used to generate a light beam, which passes through a diffraction grating causing the light to be split into three individual beams. These beams then pass through a polarized beam splitter, a quarter wavelength plate and a collimator lens before passing through a lens comprising concentric circular grooves separated by intervals of tens to hundreds of micrometres. The original collimated beam is then separated into two parts and focused onto two focal points, i.e. one to read DVD data and the other to read CD data. The beams are then reflected back along their original paths through the collimator lens and the polarized beam splitter, through a cylindrical lens, and then finally project upon a four-quadrant photodiode, which outputs a focus error signal (FES) dependent upon the distribution of the beam spot across the four quadrants. After appropriate signal processing, the FES is used to drive the VCM such that the position of 
the objective lens focal point falls on the surface of the disk and the value of the FES returns to zero. The lens ceases movement when the mechanical system attains an equilibrium condition. The current needed to maintain the objective lens steady is converted into a corresponding voltage signal which is referred to as the servo-FES, which represents the voltage required to drive the VCM. In this paper, the displacement of the VCM movement is recorded as an indication of the surface profile variations of the measured object.

\subsection{Analysis of system hysteresis}

In the present study, autofocusing is achieved by using a voice coil motor (VCM) to drive the objective lens either towards or away from the measured surface. The necessary movement is caused by the Lorentz force which results from the interaction of a magnetic field generated by passing an electrical current through a coil incorporated within the VCM and a set of permanent magnets surrounding the objective lens. It is known that magnetic materials exhibit a hysteresis phenomenon, which will influence the operation of the autofocusing system. Specifically, the hysteresis effect influences the relationship between the actual displacement of the objective lens and the driving voltage supplied to the VCM. The materials magnetized by the passage of current through the coil possess a residual magnetic moment, which generates a residual magnetic field. This provides a corresponding magnetic driving force, which must be balanced by means of the spring system shown in figure 1. As a result, there is a difference between the effective driving electrical current and the supplied electrical current, and this results in a difference in the driving voltage of the VCM. Consequently, when the system performs the autofocusing operation, it is observed that the driving voltages associated with moving the objective lens over the same range of travel either towards or away from the measured surface follow different paths.

This study employs a Sony DVD KHM-220AAA [12], which comprises a VCM with a moving range of approximately $1.4 \mathrm{~mm}$. It is noted that the displacement range associated with a positive VCM driving voltage is approximately $700 \mu \mathrm{m}$, i.e. approximately half that of the total moving range. However, the displacement characteristics of the positive voltage range mirror those of the negative voltage range. Furthermore, the measurement process generally involves operations within the positive voltage range. Consequently, the analysis of the present study is confined to a positive moving range of $600 \mu \mathrm{m}$, which deliberately excludes the final $100 \mu \mathrm{m}$ of the objective lens displacement in the forward direction in order to improve the linearity characteristics.

Figure 2 illustrates the experimental set-up of the DVD pickup head autofocusing system for hysteresis errors test. A precision micro-positioning stage developed by Anorad Co. (Model PCLM: SP4) was used to drive the pickup head at incremental steps of $5 \mu \mathrm{m}$ each time. Meanwhile, a National Instruments PC-LPM-16PNP data acquisition card and an HP5529 laser interferometer were used to record the VCM driving voltage and the corresponding objective lens displacement, respectively. The stage was moved by the PC commands in steps. The objective lens was then moved to

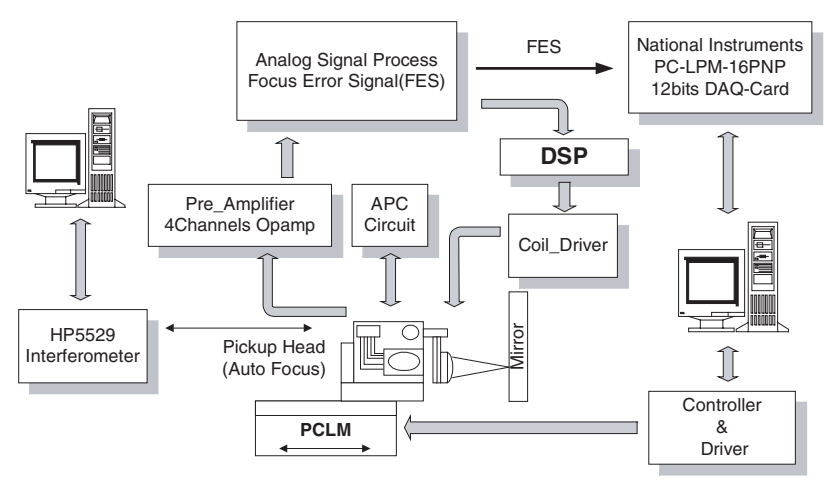

Figure 2. Experimental set-up for hysteresis analysis.

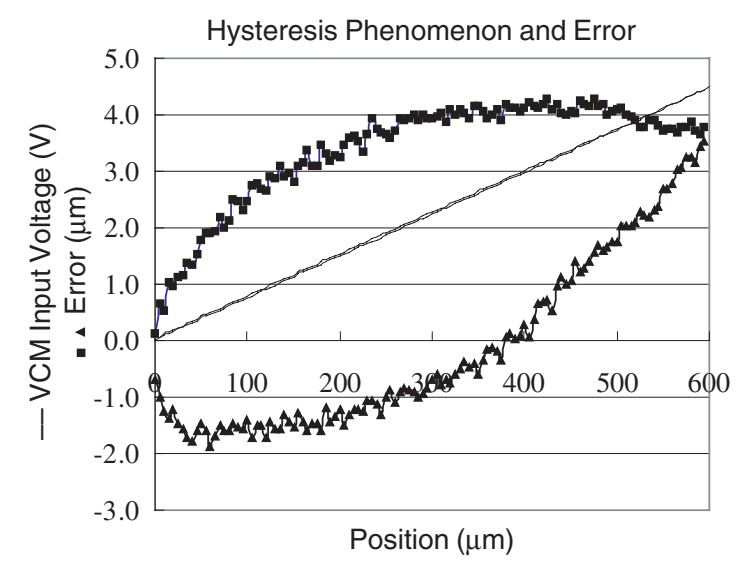

Figure 3. Hysteresis errors over the $600 \mu \mathrm{m}$ displacement range; forward error, $\boldsymbol{\Delta}$ backward error.

remain focused progressively through the displacement range of $600 \mu \mathrm{m}$ and the corresponding VCM driving voltage was noted at each step. This feedback control process was repeated until the stage returned in incremental steps to its original position.

The solid lines in figure 3 indicate the relationship between the measured values of the VCM input voltage (its scale not shown in the figure) and the objective lens displacement as it moves in both directions over $600 \mu \mathrm{m}$ range. It can be seen that although the objective lens always remains focused upon the reflecting surface as the pickup head first moves forwards and then returns backwards to its original position, the corresponding VCM input voltages exhibit slightly different paths. The forward voltage is higher than the backward voltage, which reveals the effect of hysteresis errors. It is assumed that the VCM voltage should be linear to the lens displacement, which is the same as the stage movement directly measured by the HP5529 interferometer. The actual lens motion can then be calculated directly from its linear function of VCM voltage. However, due to the hysteresis errors of the VCM voltages, the calculated displacements also show the same errors. The difference between the calculated displacement and that measured directly using the HP5529 interferometer can be clearly seen in figure 3 in which the forward and backward directions are indicated as $\boldsymbol{\square}$ and $\mathbf{\Delta}$, respectively.

From figure 3, it can be seen that the range of hysteresis error over the displacement range of $600 \mu \mathrm{m}$ is of the order of 


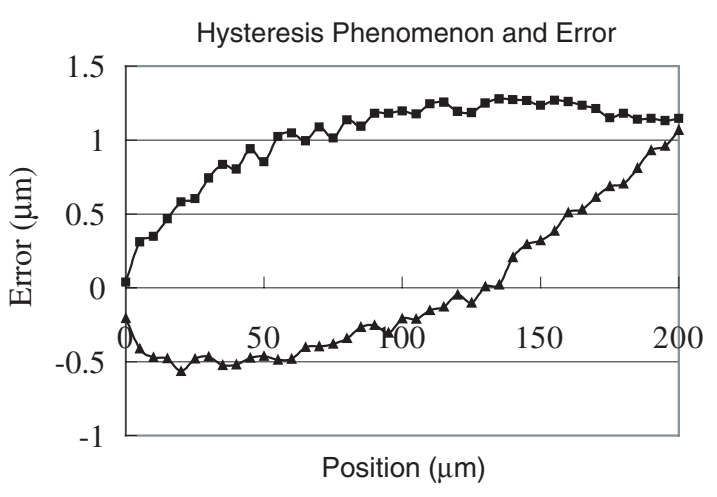

Figure 4. Hysteresis errors over the $200 \mu \mathrm{m}$ displacement range; forward error, $\boldsymbol{\Delta}$ backward error.

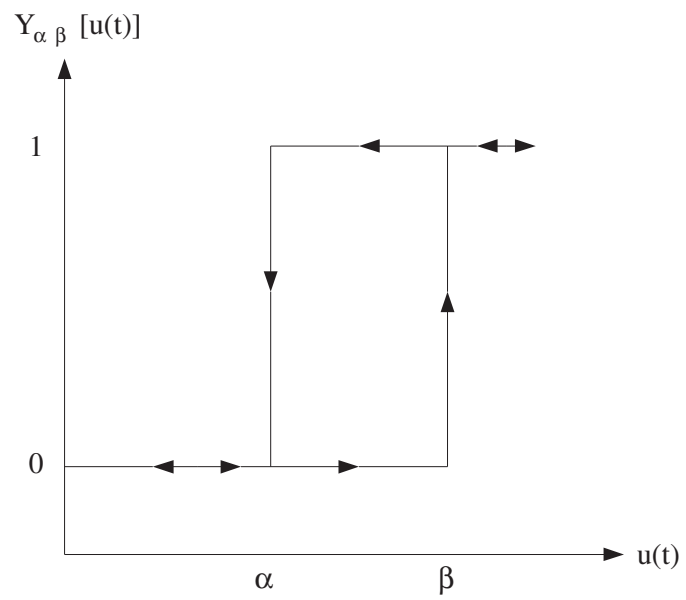

Figure 5. Single hysteresis operator.

$6.2 \mu \mathrm{m}$. In order to enable a more detailed investigation into the hysteresis phenomenon, the experiment was repeated over a restricted displacement range of $200 \mu \mathrm{m}$. A similar trend of hysteresis errors is shown in figure 4 in which the error range is reduced to approximately $1.8 \mu \mathrm{m}$.

\section{Hysteresis compensation model}

\subsection{Preisach model}

The present study adopts the Preisach model to simulate the hysteresis of the VCM and to develop a hysteresis compensation model. The Preisach model is expressed by

$$
X(t)=\iint_{\alpha \geqslant \beta} \mu(\alpha, \beta) \gamma_{\alpha \beta}[u(t)] \mathrm{d} \alpha \mathrm{d} \beta
$$

where $X(t)$ represents the hysteresis output response, $u(t)$ is the input, $\gamma_{\alpha \beta}[u(t)]$ is the hysteresis operator, $\mu(\alpha, \beta)$ is the weighting function corresponding to $\gamma_{\alpha \beta}[u(t)]$, and $\alpha$ and $\beta$ are the input $u(t)$ increase and decrease switching values, respectively.

Figure 5 illustrates the relationship between the input and the output response of a single hysteresis operator. When the value of $u(t)$ is greater than $\alpha$, the value of the hysteresis operator, $\gamma_{\alpha \beta}[u(t)]$, increases towards a value of 1 . Conversely, when the value of $u(t)$ is less than $\beta, \gamma_{\alpha \beta}[u(t)]$, decreases towards zero. The Preisach model considers the

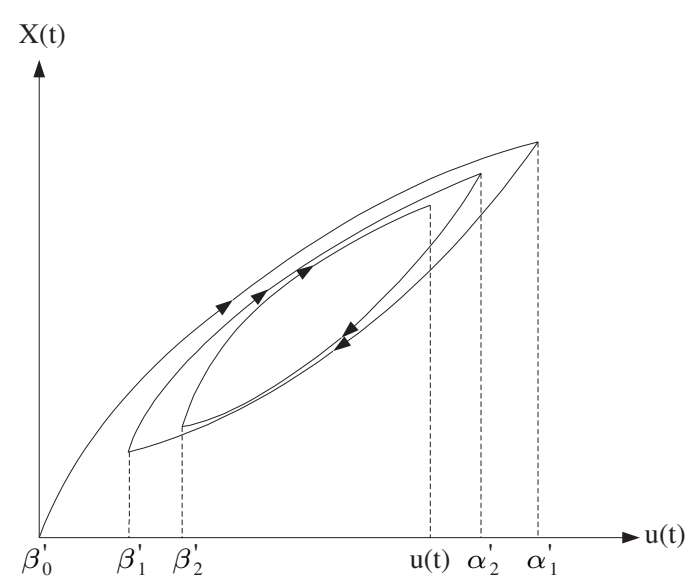

Figure 6. Hysteresis circuit for increasing input voltage.

hysteresis effect to be the combined consequence of many different individual hysteresis operators, which share the same characteristics, but which possess different values of $\alpha$ and $\beta$. Consequently, as the value of the input increases, the individual hysteresis operators do not increase to a value of 1 simultaneously. However, as the input continues to rise gradually, an increasing number of hysteresis operators do attain a value of 1 and, consequently, $X(t)$ adopts the form of the lower curve in the hysteresis circuit. Similarly, as $u(t)$ decreases, an increasing number of the hysteresis operators return to a value of 0 , in which case $X(t)$ represents the upper hysteresis curve.

By applying a series of deductions and calculations to equation (1), it can be determined that the integral of any excitation area $S\left(\alpha^{\prime}, \beta^{\prime}\right)$ can be replaced by the displacement value, $X\left(\alpha^{\prime}, \beta^{\prime}\right)$, i.e.

$$
X\left(\alpha^{\prime}, \beta^{\prime}\right)=x_{\alpha^{\prime}}-x_{\alpha^{\prime} \beta^{\prime}}=\iint_{S\left(\alpha^{\prime}, \beta^{\prime}\right)} \mu(\alpha, \beta) \mathrm{d} \alpha \mathrm{d} \beta
$$

where $X\left(\alpha^{\prime}, \beta^{\prime}\right)$ is the displacement output when the input voltage changes from $\alpha^{\prime}$ to $\beta^{\prime}, x_{\alpha^{\prime}}$ is the input position of the actuator when the input voltage increases to $\alpha^{\prime}$, and $x_{\alpha^{\prime} \beta^{\prime}}$ is the output position of the actuator when the input voltage decreases from $\alpha^{\prime}$ to $\beta^{\prime}$.

In the present practical application, equation (2) can be simplified by considering the forward and backward displacement stages separately.

(a) Forward stage:

As shown in figure 6, the input voltage increases in the final part of this stage, and equation (2) can be expressed in the following simplified form:

$$
\begin{aligned}
x(t) & =\iint_{S(t)} \mu(\alpha, \beta) \mathrm{d} \alpha \mathrm{d} \beta=\left[X\left(\alpha_{1}^{\prime}, \beta_{0}^{\prime}\right)-X\left(\alpha_{1}^{\prime}, \beta_{1}^{\prime}\right)\right] \\
& +\left[X\left(\alpha_{2}^{\prime}, \beta_{1}^{\prime}\right)-X\left(\alpha_{2}^{\prime}, \beta_{2}^{\prime}\right)\right]+X\left[u(t), \beta_{2}^{\prime}\right] \\
x(t) & =\sum_{k=1}^{N}\left[X\left(\alpha_{k}^{\prime}, \beta_{k-1}^{\prime}\right)-X\left(\alpha_{k}^{\prime}, \beta_{k}^{\prime}\right)\right]+X\left[u(t), \beta_{k}^{\prime}\right] .
\end{aligned}
$$

(b) Backward stage:

As shown in figure 7, the input voltage decreases in the final part of this stage, and equation (2) can be expressed in the 


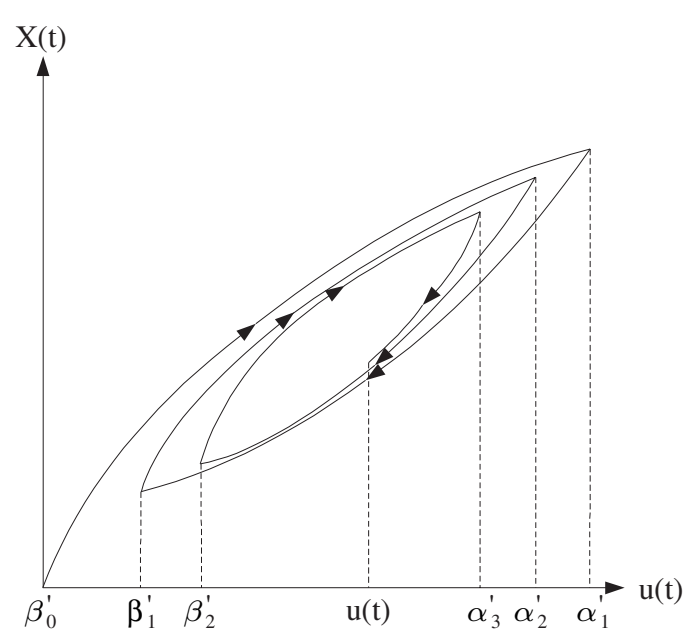

Figure 7. Hysteresis circuit for decreasing input voltage.

following simplified form:

$$
\begin{aligned}
x(t) & =\iint_{S(t)} \mu(\alpha, \beta) \mathrm{d} \alpha \mathrm{d} \beta=\left[X\left(\alpha_{1}^{\prime}, \beta_{0}^{\prime}\right)-X\left(\alpha_{1}^{\prime}, \beta_{1}^{\prime}\right)\right] \\
& +\left[X\left(\alpha_{2}^{\prime}, \beta_{1}^{\prime}\right)-X\left(\alpha_{2}^{\prime}, \beta_{2}^{\prime}\right)\right]+\left\{X\left(\alpha_{3}^{\prime}, \beta_{2}^{\prime}\right)-X\left[\alpha_{3}^{\prime}, u(t)\right]\right\} \\
x(t) & =\sum_{k=1}^{N-1}\left[X\left(\alpha_{k}^{\prime}, \beta_{k-1}^{\prime}\right)-X\left(\alpha_{k}^{\prime}, \beta_{k}^{\prime}\right)\right] \\
& +\left\{X\left(\alpha_{N}^{\prime}, \beta_{N-1}^{\prime}\right)-X\left[\alpha_{N}^{\prime}, u(t)\right]\right\} .
\end{aligned}
$$

\subsection{Hysteresis compensation}

To compensate for the hysteresis phenomenon, a computerbased data table is established in which the output displacement and corresponding input voltage are recorded at discrete steps within a series of individual maximum voltage hysteresis circuits. Subsequently, substitution of the measured VCM driving voltage into this table yields the corresponding displacement of that step. Substitution of this displacement value into equation (3) or (4) will then provide the actual profile measurement of the object. In the present study, the required table of input voltages and corresponding displacements was established by considering a total of 120 different hysteresis loops over the $600 \mu \mathrm{m}$ range with the range increment of $5 \mu \mathrm{m}$ per loop. Within each loop, the input voltage and displacement values were measured at intervals of $1 \mu \mathrm{m}$. The measurement results were then recorded in the form of the table mentioned above. Figure 8 shows the corresponding experimental data in graphical form.

Having established the table of input voltages and displacements, the current study proceeded to verify the effectiveness of the proposed compensation method. The accuracy of the compensation method was investigated by substituting the experimental data presented in figures 3 and 4 into the Preisach model, and then by comparing the calculated displacement values with the measured values. Figures 9 and 10 present the calculated error values for the displacement ranges of $600 \mu \mathrm{m}$ and $200 \mu \mathrm{m}$, respectively.

Figure 9 shows that the total range of the hysteresis error for the forward and backward paths over the displacement of $600 \mu \mathrm{m}$ decreases from the original value of $6.2 \mu \mathrm{m}$

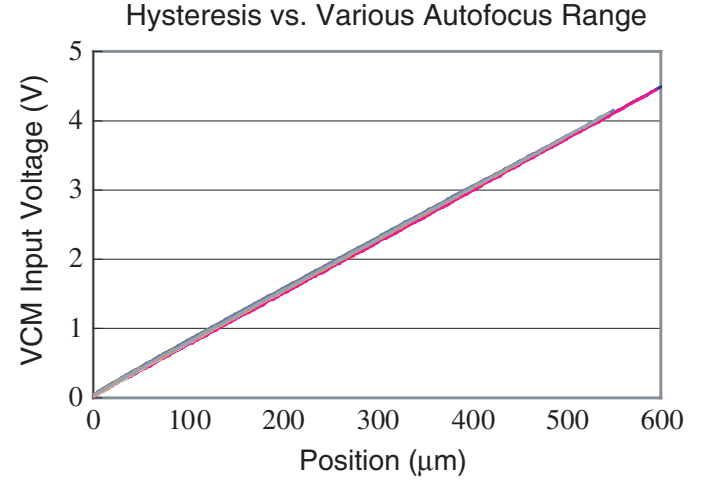

Figure 8. Experimental hysteresis results for various autofocusing ranges.

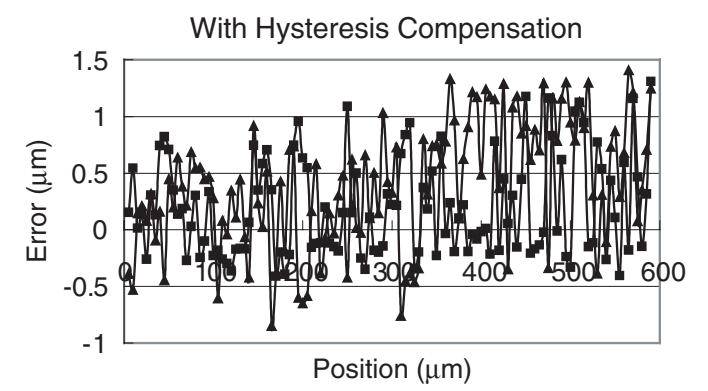

Figure 9. Error between compensated displacement and measured displacement over $600 \mu \mathrm{m}$ autofocusing range ( $\boldsymbol{\square}$ forward and $\boldsymbol{\Delta}$ backward).

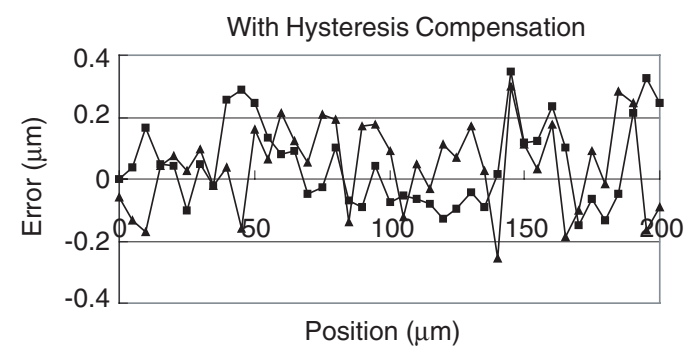

Figure 10. Error between compensated displacement and measured displacement over $200 \mu \mathrm{m}$ autofocusing range ( $\boldsymbol{\square}$ forward and $\boldsymbol{\Delta}$ backward).

to somewhere in the region of $2.2 \mu \mathrm{m}$ when the proposed hysteresis compensation method is applied. Similarly, over the restricted autofocusing displacement of $200 \mu \mathrm{m}$, figure 10 indicates that the error range falls from an original value of $1.8 \mu \mathrm{m}$ to a range of approximately $0.6 \mu \mathrm{m}$.

This experiment was conducted with the set-up as shown in figure 2. There were total 120 loops with incremental displacement range of $5 \mu \mathrm{m}$ each time. In each loop the incremental step taken for measurement was $1 \mu \mathrm{m}$. It was indeed a very time consuming task. The $1 \mu \mathrm{m}$ incremental step is the minimum command of the micro-stage. Whether the compensated errors can be further reduced if the incremental step is less than $1 \mu \mathrm{m}$ is not clear in this work. Figures 9 and 10 show the remaining random errors after compensation. It could be due to some other causes, such as the stability of the supplied voltages, the controller error of the stage, etc. The authors judge that even if the incremental step is smaller the improvement in error compensation will not be significant. 


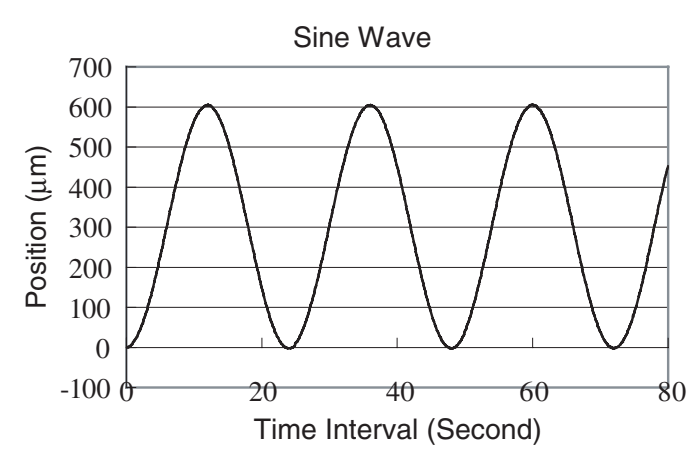

Figure 11. Sinusoidal stage motion over time.

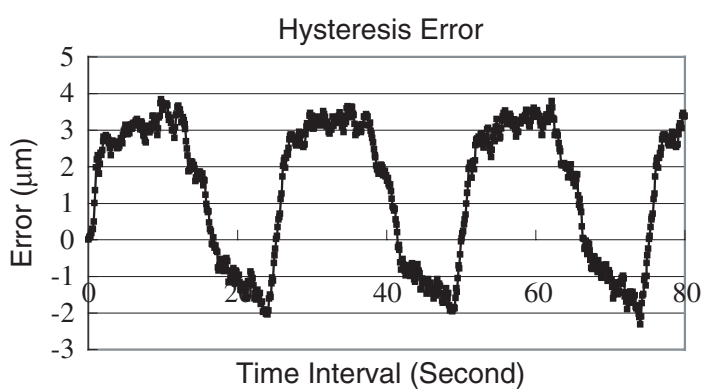

Figure 12. Uncompensated hysteresis error of VCM for sinusoidal displacement.

To investigate the effectiveness of the proposed compensation method when applied to the VCM in a real measurement case, an experimental set-up was established in which the micro-stage was first driven forwards by sinusoidal commands through a displacement of $600 \mu \mathrm{m}$ and then returned to its original position in a total cycle time of $25 \mathrm{~s}$. This cycle was repeated several times in order to generate the sine curve presented in figure 11. As the objective lens always follows the stage motion forwards and backwards to lock its focal point, the voltage driving voltage was recorded at discrete values of the displacement.

Applying the linear function of the VCM driving voltage verses objective lens displacement the corresponding displacement for any driving voltage is able to be calculated. Subtracting the calculated uncompensated displacement from the actual displacement measured using an HP5529 interferometer yields the hysteresis error. The variation in this error over time is presented in figure 12 .

Figure 12 indicates that the uncompensated hysteresis error ranges from $-2.3 \mu \mathrm{m}$ to $+4 \mu \mathrm{m}$, giving a total error range of $6.3 \mu \mathrm{m}$. As indicated in figure 3 , the forward hysteresis error is slightly larger than the backward error, a bias in the error magnitude is also shown in figure 12 in the time domain.

To investigate the effectiveness of the proposed hysteresis compensation method, the experimental data of figure 11 were substituted into the Preisach model to determine the calculated value of displacement for any driving voltage. The compensated hysteresis error is then given by the difference between the calculated displacement and the measured displacement. Figure 13 plots the compensated hysteresis error as a function of time.

Figure 13 indicates that when the compensation method is applied, the hysteresis error range extends from $+1.5 \mu \mathrm{m}$ to $-0.8 \mu \mathrm{m}$, giving a total error range of $2.3 \mu \mathrm{m}$.

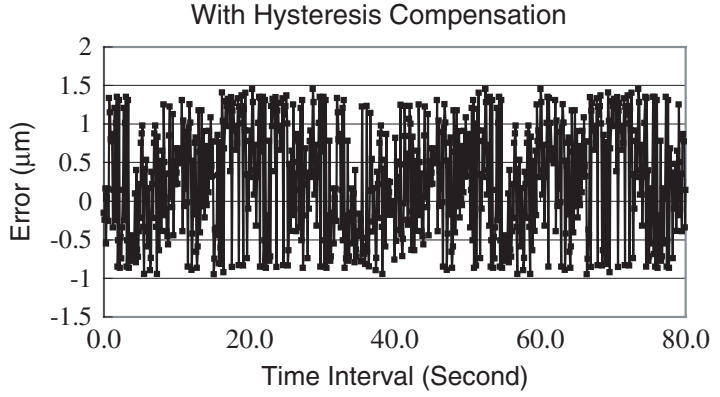

Figure 13. Compensated hysteresis error of VCM for sinusoidal displacement.

From the experimental results presented above, it can be seen that the compensation method reduces the error by more than $60 \%$, thus confirming the effectiveness of the proposed Preisach model hysteresis compensation method.

\section{Concluding remarks}

It is known that the hysteresis phenomenon will introduce measurement errors when a VCM is applied within an autofocusing laser probe measurement system. Therefore, the present study has used the Preisach model to develop a compensation method, which demonstrates the effectiveness of hysteresis error compensation scheme. The current results have shown that the proposed method reduces the range of hysteresis error from $6.2 \mu \mathrm{m}$ to less than $2.2 \mu \mathrm{m}$ over a $600 \mu \mathrm{m}$ displacement range. Furthermore, when a limited displacement range of $200 \mu \mathrm{m}$ is considered, the developed method reduces the error from $1.8 \mu \mathrm{m}$ to less than $0.6 \mu \mathrm{m}$. It demonstrates that the smaller the displacement range, the less the hysteresis error becomes. Finally, regarding the hysteresis compensation of the VCM in a real measurement case, the results have shown that the proposed compensation method reduces the error range from $6.3 \mu \mathrm{m}$ to less than $2.3 \mu \mathrm{m}$ over the VCM displacement range of $600 \mu \mathrm{m}$. An analysis of the results reveals that the compensation method reduces the hysteresis error range by approximately $60 \%$. This result clearly demonstrates the effectiveness of the proposed method.

So far, this work has achieved the linearization of bidirectional motion of the VCM pickup head through hysteresis error compensation. It is still in the stage of feed-forward control utilization hysteresis error budget. A better approach should be the real feedback control employing a displacement sensor for the actual VCM motion. Since the DVD pickup head is already a compact and miniature module it is very difficult to add a sensor to it. Future work will address this problem.

\section{References}

[1] Whitehouse D J 1988 Comparison between stylus and optical methods for measuring surfaces Ann. CIRP 37 649-53

[2] Fang-Jung Shiou 1998 Entwicklung von Strategien und Messverfahren zur Erfassung dimensioneller Bauteilgroessen fuer die Laserbearbeitung Dissertation RWTH Aachen

[3] Benschop J and Rosmalen G V 1991 Confocal compact scanning optical microscope based on compact disc technology Appl. Opt. 30 1179-84 
[4] Armstrong T R and Fitzgerald M P 1992 An autocollimator based on the laser head of a compact disc player Meas. Sci. Technol. 3 1072-6

[5] Quercioli F, Mannoni A and Tiribilli B 1997 Correlation optical velocimetry with a compact disk pickup Appl. Opt. 36 6372-5

[6] Zhang J H and Cai L L 1997 An autofocusing measurement system with a piezoelectric translator IEEE/ASME Trans. Mechatronics 2 213-6

[7] Fan K C, Lin C Y and Shyu L H 2000 Development of a low-cost focusing probe for profile measurement Meas. Sci. Technol. 11 1-7
[8] Fan K C, Chu C L and Mou J I 2001 Development of a low-cost autofocusing probe for profile measurement Meas Sci. Technol. 12 2137-46

[9] Goldfarb M and Celanovic N 1997 Modeling piezoelectric stack actuators for control of micromanipulation IEEE Control Syst. 17 69-79

[10] Donald C and Santosh D 1998 Hysteresis and vibration compensation for piezoactuators J. Guid. Control Dyn. 21 710-7

[11] Ping G and Musa J 1995 Modeling hysteresis in piezoceramic actuator Precis. Eng. 17 211-21

[12] Sony 2000 Specifications of Model KHM-210AAA 